\title{
Solution Limiters and Flux Limiters for High Order Discontinuous Galerkin Schemes.
}

\author{
Natalia Petrovskaya \\ School of Mathematics, University of Birmingham, \\ Edgbaston, B15 2TT, Birmingham, UK \\ n.b.petrovskaya@bham.ac.uk \\ www home page: http://web.mat.bham.ac.uk/N.B.Petrovskaya
}

\begin{abstract}
We analyze a general concept of limiters for a high order DG scheme written for a 1-D problem. The limiters, which are local and do not require extended stencils, are incorporated into the solution reconstruction in order to meet the requirement of monotonicity and avoid spurious solution overshoots. A limiter $\beta$ will be defined based on the solution jumps at grid interfaces. It will be shown that $\beta$ should be $0<\beta<1$ for a monotone approximate solution.
\end{abstract}

\section{Introduction.}

Recently a number of new discretization methods have been developed to numerically solve modern problems of science and engineering. One of them is a Discontinuous Galerkin (DG) discretization scheme [3], which affords optimal orders of convergence for smooth problems by using high order approximating spaces. However, the capability of high order DG schemes to resolve solution discontinuities is still an open question. It has been observed many times (e.g. see $[2,5-7])$ that a high order DG discretization may result in oscillations in the vicinity of a shock discontinuity. The study carried out in [8] has shown that high order DG approximations do not provide a monotone solution near the shock even for the simplest linear advection problem.

Since the solution oscillations may have a disastrous impact on the convergence of the approximate solution, a limiting procedure which allows one to obtain a monotone solution near discontinuities should be addressed. A number of authors have contributed to the issue of limiters for the DG scheme in recent years $[1,2,7,10]$. The examples of limiters implemented in a semi-discrete DG scheme are given in [3]. The approach suggested in [6] is similar to that in ENO schemes and takes data from neighboring grid cells to construct a local solution limiter on a given cell. The discussion of using a limiting algorithm for multi-dimensional problems can be found in [5].

In the present paper we develop an approach to define a limiter on a compact discretization stencil for high order DG schemes. We analyze one-dimensional problems where the definition of a new limiter is straightforward for a high order DG discretization. The limiter $\beta$, which is local and does not require extended 
stencils, is based on the solution jumps at grid interfaces. It will be proved that the values $0<\beta<1$ provide a monotone approximate solution over a computational grid. One important feature of the suggested approach is that we also incorporate the evaluation of flux approximation into the limiting procedure, as upwind flux approximation in a high order DG scheme presents another difficulty when steady state solutions are considered.

\section{The definition of a discontinuous Galerkin scheme.}

Our concept of limiters in high order DG schemes can be best illustrated by consideration of an ordinary differential equation written for a function $u(x)$ as

$$
F_{x}(x, u)=S(x), \quad x \in \Omega=[a, b] .
$$

The function $F(x, u(x))$ is considered as a flux function for a steady state problem (1). The equation above should be augmented with a boundary condition that will be further provided for a given problem under consideration.

We use a discontinuous Galerkin method to obtain a numerical solution to the problem. Let us introduce the element partition $G$ of the region, $G=\bigcup_{i=1}^{N} e_{i}$, $e_{i}=\left[x_{i}, x_{i+1}\right], 1 \leq i \leq N$, where $x_{i}$ is a nodal coordinate, and $h_{i}=x_{i+1}-x_{i}$ is a grid step size. We seek an approximation $u_{h}(x)$ to the solution $u(x)$ such that $u_{h}(x)$ is a piecewise polynomial function over $\Omega$. The approximate solution $u_{h}(x)$ is expanded on each grid cell as

$$
u_{h}(x)=\sum_{k=0}^{K} u_{k} \phi_{k}(x), \quad x \in e_{i}=\left[x_{i}, x_{i+1}\right]
$$

where the test functions are $\phi_{k}(x)=\left(\left(x-x_{i}\right) / h_{i}\right)^{k}, x \in e_{i}, k=0,1, \ldots, K$.

Multiplying the equation (1) by the test function $\phi_{k}(x)$ and integrating over the cell $\left[x_{i}, x_{i+1}\right]$ results in the following weak formulation of the problem,

$$
\begin{gathered}
F\left(x_{i+1}, u\left(x_{i+1}\right)\right) \phi_{k}\left(x_{i+1}\right)-F\left(x_{i}, u\left(x_{i}\right)\right) \phi_{k}\left(x_{i}\right)- \\
\int_{x_{i}}^{x_{i+1}} F(x, u)\left(d \phi_{k}(x) / d x\right) d x=\int_{x_{i}}^{x_{i+1}} S(x) \phi_{k}(x) d x, \quad k=0,1, \ldots, K
\end{gathered}
$$

where the function $u(x)$ should be further replaced by the approximate solution $u_{h}(x)$.

Since $u_{h}(x)$ is discontinuous at the cell interfaces, the above equations considered for the solution $u_{h}(x)$ require a numerical flux $\tilde{F}\left(x, u_{h}\right)$ consistent with the continuous flux $F(x, u)$ to be defined. Suppose that the flux $\tilde{F}\left(x, u_{h}\right)$ that generally depends on the two values of the approximate solution at any grid 
interface $x_{i}$ is chosen for a given problem (see [4] for the discussion of numerical fluxes). Then the DG discretization scheme reads

$$
\begin{gathered}
\tilde{F}\left(u_{h}\left(x_{i+1}\right)\right) \phi_{k}\left(x_{i+1}\right)-\tilde{F}\left(u_{h}\left(x_{i}\right)\right) \phi_{k}\left(x_{i}\right)- \\
\int_{x_{i}}^{x_{i+1}} F\left(x, u_{h}(x)\right)\left(d \phi_{k}(x) / d x\right) d x=\int_{x_{i}}^{x_{i+1}} S(x) \phi_{k}(x) d x, \quad k=0,1, \ldots, K .
\end{gathered}
$$

Although very efficient for smooth problems, a high order DG discretization is not always appropriate when discontinuous functions are concerned. It has been shown in [8] that being applied to a discontinuous problem the DG scheme (4) may generate solution overshoots that do not depend on the grid step size. Below we address the issue of limiters required to eliminate spurious solution oscillations in a high order DG scheme.

\section{Limiters for a high order DG scheme.}

Let $U(x)$ be the exact solution to the boundary-value problem for the equation (1) and three points $P_{i}=\left(x_{i}, U\left(x_{i}\right)\right), P=(\hat{x}, U(\hat{x}))$, and $P_{i+1}=\left(x_{i+1}, U\left(x_{i+1}\right)\right)$ be chosen at the curve $U(x)$ (see Fig. $1 a$ ). Let the distance $x_{i+1}-x_{i}=h>0$, and we denote $\left(\hat{x}-x_{i}\right) /\left(x_{i+1}-x_{i}\right)=s_{0}, s_{0} \in(0,1)$. We define the parameter $\theta$ as follows

$$
\theta=\left(U(\hat{x})-U\left(x_{i}\right)\right) /\left(U\left(x_{i+1}\right)-U\left(x_{i}\right)\right), \quad U\left(x_{i+1}\right) \neq U\left(x_{i}\right),
$$

Let us fix $s_{0}$ and move the points $P$ and $P_{i+1}$ along the curve $U(x)$. The parameter $\theta$ is then considered as a function of the distance $h$.

The behavior of $\theta(h)$ depends on the solution $U(x)$. Let $\mathrm{U}(\mathrm{x})$ be a monotone function shown in Fig. 1a. For the monotone solution, the following conditions hold

1. $\left|U(\hat{x})-U\left(x_{i}\right)\right|<\left|U\left(x_{i+1}\right)-U\left(x_{i}\right)\right|$, if $\hat{x}-x_{i}<x_{i+1}-x_{i}$,

2. $\operatorname{sgn}\left(U(\hat{x})-U\left(x_{i}\right)\right)=\operatorname{sgn}\left(U\left(x_{i+1}\right)-U\left(x_{i}\right)\right)$.

Hence, $\theta(h)>0$, and $\theta(h)$ is a bounded function over the domain of definition.

We now estimate the value of $\theta$ for $h \rightarrow 0$. The solution is assumed to be a smooth function over the interval $\left[x_{i}, x_{i+1}\right]$. We denote the $k-t h$ derivative $d^{(k)} U(x) / d x^{k}$ taken at the point $x_{i}$ as $D_{k}$. The Taylor series expansion of the solution $U(x)$ near the point $x_{i}$ yields $U(\hat{x}) \approx U\left(x_{i}\right)+D_{1} s_{0} h$, and

$$
\begin{aligned}
& 1 /\left(U\left(x_{i+1}\right)-U\left(x_{i}\right)\right) \approx 1 /\left(D_{1} h+(1 / 2) D_{2} h^{2}\right)=1 /\left(D_{1} h\left(1+\left(D_{2} / 2 D_{1}\right) h\right)\right) \approx \\
& 1 /\left(D_{1} h\left(1-\left(D_{2} / 2 D_{1}\right) h\right)\right), \quad D_{1} \neq 0 .
\end{aligned}
$$

Substituting the expansion above into (5), we obtain

$$
\theta \approx\left(D_{1} s_{0} h\left(1-\left(D_{2} / 2 D_{1}\right) h\right)\right) / D_{1} h=s_{0}(1-O(h)) .
$$

For the sake of simplicity, let us further consider $x_{i}=0$, so that $x_{i+1}=h$. There are three extreme cases of a monotone smooth function $U(x)$ that define the behavior of $\theta(h)$ : 

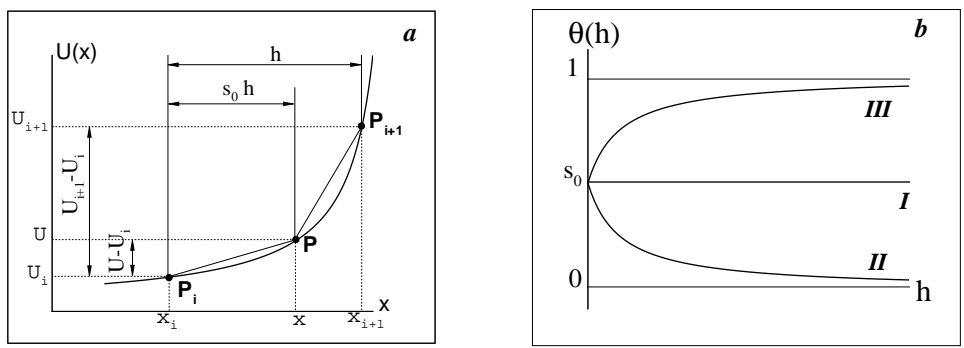

Fig. 1. A monotone solution $U(x)$. (a) Geometric interpretation of the function $\theta(h)$ in the $(x, U(x))$ - plane. (b) The function $\theta(h)$ for a monotone solution $U(x)$ : (I) a linear function $U(x)$, (II) a concave function $U(x)$, (III) a convex function $U(x)$.

1. $U(x)$ is a linear function. The definition of $\theta$ yields $\theta(h)=$ const $=s_{0}$. The function $\theta(h)$ is presented by curve $(I)$ in Fig. $1 b$.

2. $U(x)$ is a concave function, which has a vertical asymptote at the point $x=h^{*}: U(x) \rightarrow \infty$, as $x \rightarrow h^{*}$. Since $|U(h)-U(0)| \rightarrow \infty$, and $\mid U\left(s_{0} h\right)-$ $U(0) \mid \rightarrow \Delta U \neq \infty$, as $x \rightarrow h^{*}$, we have $\theta(h) \rightarrow 0$, as $h \rightarrow h^{*}$. If we consider a set of smooth concave functions, then $h^{*} \rightarrow \infty$, and we arrive at $\theta(h) \rightarrow 0$, as $h \rightarrow \infty$. The function $\theta(h)$ for a concave $U(x)$ is shown as curve $(I I)$ in Fig. $1 b$.

3. $U(x)$ is a convex function, which has a horizontal asymptote: $U(x) \rightarrow U_{0}$, as $x \rightarrow \infty$. Since $U(h)-U(0) \rightarrow U_{0}-U(0)$, and $U\left(s_{0} h\right)-U(0) \rightarrow U_{0}-U(0)$, as $x \rightarrow \infty$, we have $\theta(h) \rightarrow 1$, as $h \rightarrow \infty$. The function $\theta(h)$ generated by a convex $U(x)$ is shown as curve $(I I I)$ in Fig. $1 b$.

Hence, for a monotone smooth function $U(x)$, the parameter $\theta$ is bounded by $0<\theta \leq 1$, where $\theta=1$ for $U(x) \equiv$ const by convention.

We now consider a non-monotone solution $U(x)$ shown in Fig. $2 a$. Let $P_{\text {ext }}=$ $\left(x_{\text {ext }}, U\left(x_{\text {ext }}\right)\right)$ be an extremum point. For small $h<x_{\text {ext }}$, the solution $U(x)$ is a monotone function and we refer to the analysis above, as the function $\theta(h)$ will depend entirely on the derivative $d^{2} U(x) / d x^{2}$. For $h>x_{e x t}$, the function $\theta(h)$ is an increasing function which takes the value $\theta\left(h_{0}\right)=1$ at the point $h=h_{0}$, where $U(h)=U\left(s_{0} h\right)$ (see Fig. 2a). The function $\theta(h)$ has a singular point $h=h_{d}$, defined by the condition $U\left(h_{d}\right)=U\left(x_{i}\right)$. Since $x_{i}=0$ and $U(h)-U(0)$ changes the sign at the point $x=h_{d}$, the asymptotic behavior of $\theta(h)$ is $\theta(h) \rightarrow+\infty$ as $h \rightarrow h_{d}-0$, and $\theta(h) \rightarrow-\infty$, as $h \rightarrow h_{d}+0$. Finally, $\theta(h) \rightarrow 0$ (or another constant), as $h \rightarrow \infty$, provided there are no other extremum points. The function $\theta(h)$ for a non-monotone solution is shown in Fig. $2 b$.

The above consideration reveals how a limiting procedure can be defined for an approximate solution in a high order DG scheme. Let $\hat{x}$ be an arbitrary point in the cell $\left[x_{i}, x_{i+1}\right]$. Suppose that the approximate solution $u_{h}(x)$ coincides with the exact solution $U(x)$ everywhere except for the point $\hat{x}$, so that a local extremum appears at $\hat{x}$ (see Fig. $3 a$ ). Since for a monotone function the condition $0<\theta \leq 1$ always holds, we now compare the approximate solution variation $\left(u_{h}(\hat{x})-u_{h}\left(x_{i}\right)\right)$ with the exact solution variation $\left(U\left(x_{i+1}\right)-U\left(x_{i}\right)\right)$. The value 

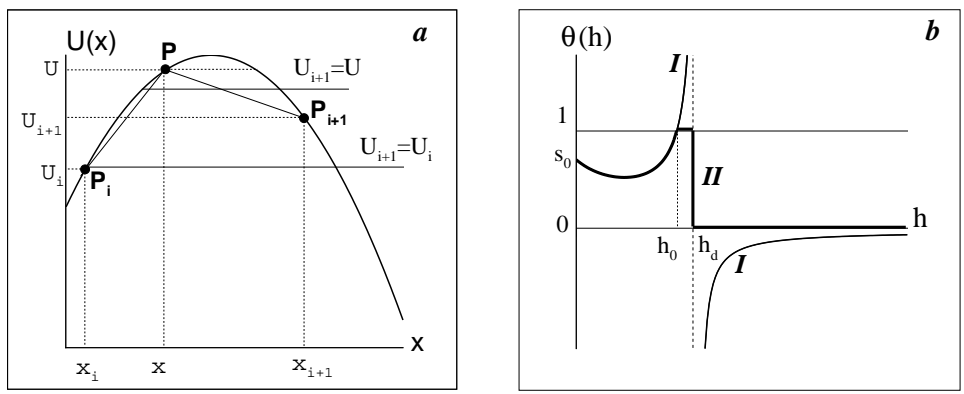

Fig. 2. A non-monotone solution $U(x)$. (a) Geometric interpretation of the function $\theta(h)$ in the $(x, U(x))$ - plane. (b) The function $\theta(h)$ for a non-monotone solution: (I) $\theta(h)$ is not bounded near the extremum point, (II) A limiting procedure cuts off $\theta(h)$ near the solution extremum.

$\theta_{i}<0$ or $\theta_{i}>1$ of the parameter

$$
\theta_{i}=\left(u_{h}(\hat{x})-u_{h}\left(x_{i}\right)\right) /\left(U\left(x_{i+1}\right)-U\left(x_{i}\right)\right)
$$

indicates that a local extremum is present in the cell $e_{i}$. The limiting procedure for a non-monotone function is shown in Fig. $2 b$ in the $(h, \theta)$-plane.

\section{The flux control in the limiting procedure.}

The limiter (7) is not viable, unless an accurate estimate of the exact solution has been given at the points $x_{i}$ and $x_{i+1}$. Hence, our next purpose is to obtain a reliable solution estimate to be used in the limiter $\theta_{i}$. Moreover, we also want the evaluation of flux approximation to be incorporated into the limiting procedure, as upwind flux approximation in a high order DG scheme presents another difficulty when steady state solutions are considered. It has been recently shown in [9] that a high order DG scheme is not able to recognize flux extrema that may lead to an underdetermined system of algebraic equations obtained as a result of the discretization. Solving that system of equation will inevitably result in an oscillating numerical solution, so that a flux control procedure should be developed to avoid a divergent solution (see [9] for a further discussion of spurious oscillations arising as a result of incorrect flux approximation).

Our approach to the flux control in the DG scheme (4) is based on the definition of "frame" and "phantom" points on a grid cell. Let $P=\left(x_{i}, u_{h}\left(x_{i}\right)\right)$ be a point in the $(x, u)$ - plane, where $x_{i} \in G$. Each pair $\left(x_{i}, u_{h}\left(x_{i}\right)\right)$ generates the flux $F\left(x_{i}, u_{h}\left(x_{i}\right)\right)$. We will refer to the point $P$ as a "frame" point and denote it as $P_{\mathcal{F}}$, if $P$ is involved into the definition of the numerical flux, i.e. $\tilde{F}\left(x_{i}, u_{h}\right)=F(P)$. Otherwise, we will refer to the point $P$ as a "phantom" point and will use the notation $P_{\mathcal{P}}$ for it. A solution estimate we use is based on the 
assumption that a discrete conservation law

$$
\tilde{F}\left(x_{i+1}, u_{h}\right)-\tilde{F}\left(x_{i}, u_{h}\right)=\int_{x_{i}}^{x_{i+1}} S(x) \phi_{k}(x) d x,
$$

is consistent with the equation (1), that is $u_{\mathcal{F}}\left(x_{\mathcal{F}}\right) \rightarrow U\left(x_{\mathcal{F}}\right), \quad h \rightarrow 0$. In other words, we assume that local extrema which do not vanish on fine grids may only appear at "phantom" points. The above requirement is to guarantee that the correct flux approximation is used in the problem.

Based on the assumption above, we suggest the following approximation to the function (7)

$$
\beta_{i}=\delta u_{i} / \Delta u,
$$

where $\delta u_{i}=u_{h}\left(x_{i+1}\right)-u_{h}\left(x_{i}\right)$ for approximate solution $u_{h}(x)$ defined at the interval $e_{i}$. The exact solution variation $\Delta U=U\left(x_{i+1}\right)-U\left(x_{i}\right)$ on the cell $e_{i}$ is replaced in limiter $\beta_{i}$ with the approximate solution variation $\Delta u=u_{h \mathcal{F}}\left(x_{i+1}\right)-$ $u_{h \mathcal{F}}\left(x_{i}\right)$ at the "frame" points.

In order to incorporate the limiter (9) into the DG scheme, the "frame" points should be defined for a given grid cell. Consider an upwind flux approximation that requires one value of the approximate solution at each grid interface for a monotone solution function. Let the "frame" points for the upwind flux be defined as $P_{\mathcal{F}_{1}}=\left(x_{i}, u_{i-1}+\delta u_{i-1}\right)$, and $P_{\mathcal{F}_{2}}=\left(x_{i+1}, u_{i}+\delta u_{i}\right)$ on the cell $e_{i}$. The solution variation is

$\Delta u=u_{i}+\delta u_{i}-u_{i-1}-\delta u_{i-1}=u_{i}+\delta u_{i}-\left(u_{i}-[u]_{i}\right)=\delta u_{i}+[u]_{i}, \quad \beta_{i}=\frac{\delta u_{i}}{\delta u_{i}+[u]_{i}}$,

where $[u]_{i}=u_{h}\left(x_{i}+0\right)-u_{h}\left(x_{i}-0\right)=u_{i}-\left(u_{i-1}+\delta u_{i-1}\right)$ is a solution jump at the interface $x_{i}$.

If the "frame" points are defined as $P_{\mathcal{F}_{1}}=\left(x_{i}, u_{i}\right)$, and $P_{\mathcal{F}_{2}}=\left(x_{i+1}, u_{i+1}\right)$, the solution variation will be

$$
\Delta u=u_{i+1}-u_{i}=\left(u_{i}+\delta u_{i}\right)+[u]_{i+1}-u_{i}=\delta u_{i}+[u]_{i+1}, \quad \beta_{i}=\frac{\delta u_{i}}{\delta u_{i}+[u]_{i+1}},
$$

where the jump $[u]_{i+1}=u_{h}\left(x_{i+1}+0\right)-u_{h}\left(x_{i+1}-0\right)=u_{i+1}-\left(u_{i}+\delta u_{i}\right)$ is considered at the interface $x_{i+1}$. Generally, the limiter $\beta_{i}$ can be written as

$$
\beta_{i}=1-\frac{[u]_{\mathcal{P}}}{\delta u_{i}+[u]_{\mathcal{P}}}
$$

so that $\beta_{i}$ depends always on the solution jump $[u]_{\mathcal{P}}$ calculated at the cell interface where a "phantom" point presents.

The limiting procedure is illustrated in Fig. $3 b$ for a piecewise linear $(K=1)$ DG discretization. The "phantom" point $u_{i}+\delta u_{i}$ is shown as a white dot, the frame points are shown as black dots. The location $(I)$ of the "phantom" point yields a monotone approximate solution, while the locations $(I I)$ and $(I I I)$ 

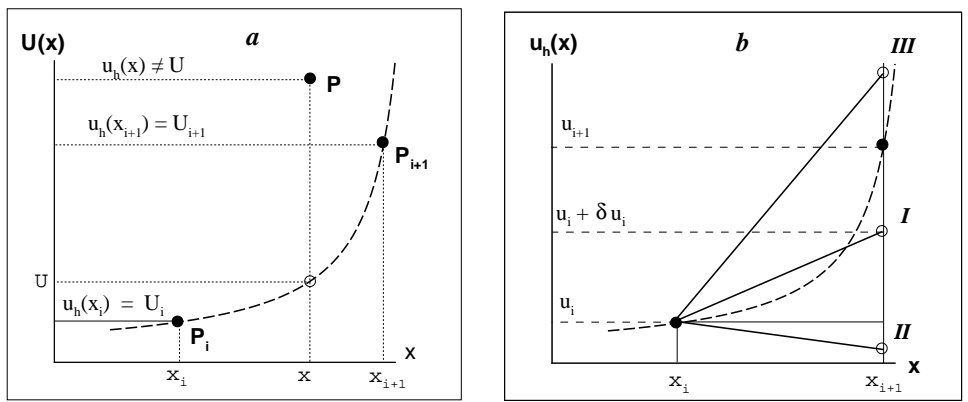

Fig. 3. Limiters for a piecewise linear approximate solution. (a) The limiter detects a solution overshoot at the interior point of the domain. (b) The limiter detects a solution overshoot at the cell interface. The "phantom" point (I) yields a monotone approximate solution. The "phantom" point (II) yields an overshoot which is indicated by $\beta_{i}<0$. The "phantom" point (III) yields an overshoot which is indicated by $\beta_{i}>1$.

result in a nonphysical local extremum. The limiter (10) detects both cases of the solution overshoot, as $\beta_{i}<0$ for the location $(I I)$ and $\beta_{i}>1$ for the location $(I I I)$.

We first illustrate the use of limiters by a simple numerical test discussed earlier in [8]. Consider the following linear boundary-value problem

$$
u_{x}=S(x), \quad u(0)=U_{0}, \quad x \in \Omega=[0,2],
$$

so that flux function $F(x, u) \equiv u$. Let a discontinuous solution $\mathrm{U}(\mathrm{x})$ to the equation (11) be given by

$$
U(x)=\left\{\begin{array}{cc}
1 .-\sqrt{0.5-x} & 0 \leq x<0.5 \\
1 & 0.5<x \leq 1 \\
\tanh (200(x-1.5)) & 1<x \leq 2
\end{array}\right.
$$

Given the solution (12), the source function $S(x)$ is reconstructed from the equation (11). The boundary condition is $u(0)=U(0)$.

For the advection equation the upwind numerical flux is $\tilde{F}\left(u_{i}, u_{i+1}\right)=u_{i}$, and we have $[u]_{\mathcal{P}}=[u]_{i}$ on any grid cell. The approximate solution obtained as a result of the DG discretization with a piecewise linear solution reconstruction is shown in Fig. $4 a$. The number of grid nodes is $N=32$.

The DG scheme (4) employed in the problem generates oscillations near the shock. Those oscillations do not vanish on fine grids, so that the limiting is required to eliminate them. Thus, we compute the limiter (10) on each grid cell. In case that $\beta<0$ or $\beta>1$ ( a solution overshoot) the solution interpolation between two "frame" points is used to obtain a monotone approximate solution.

After the limiting procedure is applied, the new solution reconstruction is shown in Fig. $4 b$. It can be seen from the figure that the new solution has no overshoots over the domain.

We now consider the inviscid Burgers' equation

$$
\frac{\partial u}{\partial t}+u \frac{\partial u}{\partial x}=0
$$



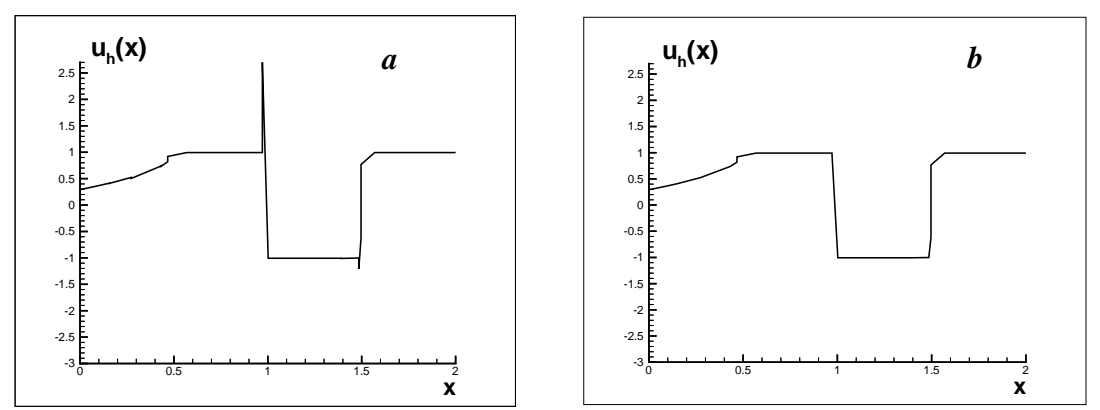

Fig. 4. The advection test problem (1) with a shock discontinuity. (a) Oscillations in the vicinity of the shock for the $D G K=1$ solution. The number of grid nodes $N=32$. (b) The solution on the same grid after the limiting procedure.

This is a well known example of a nonlinear hyperbolic equation with a quadratic flux function $F(u)=u^{2} / 2$. We solve the equation (13) in the domain $x \in[0,1]$ due to a periodic boundary condition. The initial condition has been chosen as a sine wave function

$$
u(x, 0)=u_{0}(x)=0.25+0.5 \sin (\pi(2 x-1)) .
$$

The exact solution is smooth for any time $t<1 / \pi$, while the solution becomes discontinuous at later times.

We are interested in the numerical solution to the problem (13), (14). A high order DG discretization is implemented, and we apply the Godunov flux $\tilde{F}^{G}\left(u_{l}, u_{r}\right)$ in order to discretize the function $F(u)$. The flux approximation is defined as

$$
\tilde{F}^{G}\left(u_{l}, u_{r}\right)= \begin{cases}\min _{u_{l} \leq u \leq u_{r}} F(u), & \text { if } u_{l} \leq u_{r} \\ \max _{u_{l} \leq u \leq u_{r}} F(u), & \text { otherwise }\end{cases}
$$

for the left state $u_{l}$ and right state $u_{r}$ at a given grid interface.

For numerical solution of the conservation law (13), a DG discretization in space is combined with an implicit time integration scheme. Let us notice that while the limiting procedure for the explicit Runge-Kutta integration has been introduced in the work [2] and further investigated in [5] and other works, limiters for implicit integration schemes have not been intensively discussed in literature. Thus we use a backward Euler time integration scheme in our problem to see how a suggested limiting algorithm will work for time dependent problems.

An approximate solution at $t=0.47$ is shown in Fig. 5a for a piecewise linear DG discretization on a uniform grid of 128 cells. It can be seen from the figure that the approximate solution oscillates near the shock and we need to apply the limiting procedure (10) at each time step to obtain a non-oscillating solution. A new approximate solution is shown in Fig. 5b. The limiters eliminate spurious oscillations while remaining the solution piecewise linear in the vicinity of the shock. However, further numerical validation of the limiting procedure for 

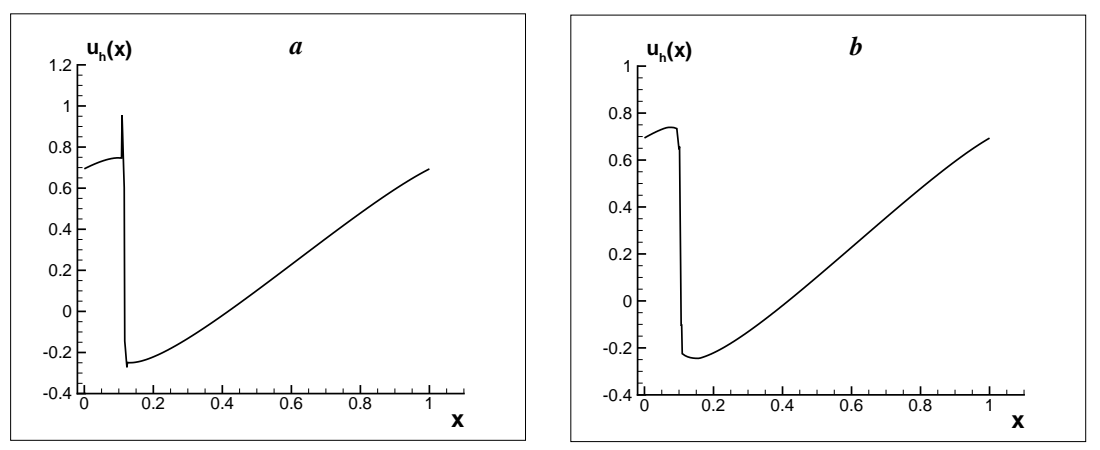

Fig. 5. The numerical solution to the inviscid Burgers' equation (13). (a) The approximate $D G K=1$ solution to the problem (13), (14) oscillates near the shock soon after the shock formation ( time $t=0.47$ ). The number of grid nodes $N=128$. (b) The solution on the same grid after the limiting procedure.

nonlinear equations is required to confirm that the suggested algorithm keeps the order of approximation in a high order DG discretization scheme. That should be considered as a topic for future work.

\section{References}

1. Burbeau, A. et al.: A Problem - Independent Limiter for High Order Runge-Kutta Discontinuous Galerkin Methods. J. Comput. Phys. 169 (2001) 111-150

2. B.Cockburn. Discontinuous Galerkin Methods for Convection - Dominated Problems, in High-Order Discretization Methods in Computational Physics, T.Barth and H.Deconinck, eds., Lecture Notes in Comput.Sci.Engrg., 9, Springer-Verlag, Heidelberg, 1999, pp.69-224.

3. Cockburn, B., Shu, C.: TVB Runge-Kutta Local Projection Discontinuous Galerkin Finite Element Method for Conservation Laws II: General Framework. Mathemat. Comput. 52 (1989) 411-435

4. Hirsh, C.: Numerical Computation of Internal and External Flows. John Wiley \& Sons. (1990)

5. Hoteit, H. et al:: New Two-Dimensional Slope Limiters for Discontinuous Galerkin Methods on Arbitrary Meshes. INRIA Rennes. 4491 (2002)

6. Huynh, H.T.: Accurate upwind methods for the Euler Equations. AIAA 95-1737 (1995)

7. Lowrier, R.B.: Compact Higher-Order Numerical Methods for Hyperbolic Conservation Laws. PhD thesis, The University of Michigan. (1996)

8. Petrovskaya, N.B.: Two Types of Solution Overshoots in Discontinuous Galerkin Discretization Schemes. Comm. Math. Sci., 3(2) (2005) 233-247

9. Petrovskaya, N.B.: On Oscillations in Discontinuous Galerkin Discretization Schemes for Steady State Problems. SIAM J. Sci. Comput. 27(4) (2006) 1329-1346

10. Tu, S., Aliabadi, S.: A Slope Limiting Procedure in Discontinuous Galerkin Finite Element Method for Gasdynamic Applications. Int. J. Numer. Anal. Model. 2(2) (2005) 163-178 